\title{
Interaction of free charged particles with a chirped electromagnetic pulse
}

\author{
A. G. Khachatryan, F. A. van Goor, and K.-J. Boller \\ Department of Applied Science, University of Twente, P.O. Box 217, 7500 AE Enschede, The Netherlands \\ (Received 13 February 2004; published 2 December 2004)
}

\begin{abstract}
We study the effect of chirp on electromagnetic (EM) pulse interaction with a charged particle. Both the one-dimensional (1D) and 3D cases are considered. It is found that, in contrast to the case of a nonchirped pulse, the charged particle energy can be changed after the interaction with a 1D EM chirped pulse. Different types of chirp and pulse envelopes are considered. In the case of small chirp, an analytical expression is found for arbitrary temporal profiles of the chirp and the pulse envelope. In the 3D case, the interaction with a chirped pulse results in a polarization-dependent scattering of charged particles.
\end{abstract}

DOI: 10.1103/PhysRevE.70.067601

PACS number(s): 41.20.-q, 42.50.Vk, 41.75.Jv

In the field of an electromagnetic (EM) pulse, a free charged particle experiences the ponderomotive force directed to regions of lower intensity [1]. However, the overall interaction of a free charged particle with a pulse of a onedimensional (1D, plane-wave) EM field does not lead to a change in the particle's energy. Any acceleration in the rising edge of the pulse is compensated for by deceleration in the trailing edge, unless the particle is "born" inside the pulse, for example due to tunneling ionization [2]. The situation is different when using an EM pulse, the field envelope of which varies in at least one transverse direction, such as, for example, in the waist of a focused Gaussian laser beam. Here, an electron can gain energy if it leaves the pulse in the transverse direction before the decelerating field compensates the acquired energy [3]. Such interaction of electrons with a laser pulse has been observed experimentally by focusing an intense laser pulse (with a peak intensity of $I_{0}$ $\approx 10^{19} \mathrm{~W} / \mathrm{cm}^{2}$ ) onto free electrons in vacuum [4].

In this article, we predict that the energy of a charged particle can be changed through the interaction with a chirped electromagnetic pulse, i.e., in which the local (instantaneous) frequency changes over the length of the pulse, even in 1D. We show that the energy gain of the particle increases with the EM pulse amplitude and with the relative chirp. Presently, high-intensity $\left(I_{0} \sim 10^{19} \mathrm{~W} / \mathrm{cm}^{2}\right)$ laser pulses with a relative chirp of a few percent are available from infrared solid-state laser systems [5] and up to a few tens percent should be possible with existing few-cycle systems [6] or after propagation of an intense fs laser pulse through a plasma channel [7]. Generation of a subpicosecond EM pulse with more than $10 \%$ chirp from a free-electron laser was demonstrated experimentally [8]. To generate even larger chirps, the reflection of EM pulses from a relativistic ionization front [9] looks promising when the gamma factor of the front changes during the reflection [10]. In the following, we investigate the interaction of an electron with a chirped EM pulse. Note, however, that our predictions remain valid also for any other type of charged particle, if one simply replaces the electric charge and the mass of the particle. We start with a $1 \mathrm{D}$ theory and then present $3 \mathrm{D}$ results.

First, consider a $1 \mathrm{D}$ chirped pulse propagating in the $Z$ (longitudinal) direction, and suppose that the field is linearly polarized in the $x$ direction, such that $E_{x}$ and $B_{y}$ are the only nonzero components of the electric field $\mathbf{E}$ and the magnetic field $\mathbf{B}$. The pulse can be described by $\mathbf{E}=\mathbf{e}_{x} E_{x}$ $=\mathbf{e}_{x} E_{0}(\zeta) \cos [\omega(\zeta) \zeta]$ and $\mathbf{B}=\mathbf{e}_{y} B_{y}=\mathbf{e}_{y} E_{x}$, where $\zeta \equiv Z / c-t$ is the retarded time, and $\omega(\zeta)$ is the local frequency in the pulse. Note that the 1D model is valid also in the threedimensional case when the particle's position remains close to the pulse axis, so that the decrease of the EM field in the transverse direction can be neglected. Next, consider a free electron with an initial momentum $\mathbf{P}_{0}\left(P_{0 x}, P_{0 y}, P_{0 z}\right)$. To study the electron's dynamics, we write the relativistic equation of motion of an electron in an EM field,

$$
d \mathbf{p} / d \tau=d(\gamma \boldsymbol{\beta}) / d \tau=-\mathbf{E}-\boldsymbol{\beta} \times \mathbf{B} .
$$

Here $\mathbf{p}=\mathbf{P} / m_{e} c$ and $\boldsymbol{\beta}=\mathbf{v} / c$ are the dimensionless momentum and velocity of the electron, $\gamma=\left(1+\mathbf{p}^{2}\right)^{1 / 2}$ is the relativistic factor, $m_{e}$ is the electron mass, and $c$ is the speed of light in vacuum, $\tau=\omega_{0} t$, and the spatial coordinates $x, y$, and $z$ are normalized to $c / \omega_{0}$, where $\omega_{0}=\omega(\zeta=0)$ is the local frequency in the center of the pulse; the variation of the dimensionless temporal (spatial) variables by $2 \pi$ corresponds to the wave period (wavelength) in the center of the pulse. The electric and magnetic fields are normalized to $m_{e} c \omega_{0} / e$, where $e$ is the absolute charge of the electron. Multiplying Eq. (1) by $\boldsymbol{\beta}$, we obtain an equation for the energy of the particle,

$$
d \gamma / d \tau=-\boldsymbol{\beta} \cdot \mathbf{E} .
$$

Note that Eqs. (1) and (2) are also valid in the field of a 3D EM pulse. From Eq. (1), in the 1D case, we have

$$
\begin{gathered}
d p_{x} / d \tau=-\left(1-\beta_{z}\right) E_{x}, \\
d p_{y} / d \tau=0, \\
d p_{z} / d \tau=-\beta_{x} E_{x} .
\end{gathered}
$$

According to Eq. (4), the $y$ component of the momentum is conserved, i.e., $p_{y}(\tau)=p_{y}(\tau=0) \equiv p_{y 0}$. From Eqs. (2) and (5), we find the following integral of motion (see, e.g., Ref. [11]):

$$
\gamma(\tau)-p_{z}(\tau)=\gamma_{0}-p_{z 0} \equiv C=\text { const. }
$$

To obtain the transverse momentum, we integrate Eq. (3), 


$$
p_{x}=p_{x 0}+A
$$

where $A$ is defined as

$$
A \equiv \int_{\xi_{0}}^{\xi} E_{x}(\xi) d \xi
$$

with $\xi=\omega_{0} \zeta=z-\tau$ and $\xi_{0}=\xi(\tau=0)$. One can see that the value of $A$ is equal to the difference of the normalized vectorpotential component $A_{x}$, defined as $E_{x}=-\partial A_{x} / \partial \tau$, in the points $\xi$ and $\xi_{0}$. Thus, Eq. (7) shows the well-known fact that the transverse canonical momentum $p_{x}-A_{x}$ is conserved. Once $p_{x}$ is calculated from Eq. (7), one can easily find the longitudinal momentum $p_{z}$ and the relativistic factor from Eq. (6),

$$
\left(\begin{array}{c}
p_{z} \\
\gamma
\end{array}\right)=\left(\begin{array}{c}
p_{z 0} \\
\gamma_{0}
\end{array}\right)+f\left(A, \vec{p}_{0}\right)
$$

where $f=A\left(A+2 p_{x 0}\right) / 2 C$. When the electron is initially nonrelativistic, $\left|\mathbf{p}_{0}\right| \ll 1$, one obtains $C \simeq 1$ and thus $f=A^{2} / 2$ $+A p_{x 0}$. For an initially relativistic electron with the longitudinal momentum prevailing, $\left(p_{x 0}\right)^{2}, \quad\left(p_{y 0}\right)^{2} \ll\left(p_{y 0}\right)^{2} \gg 1$, we have from Eq. (9)

$$
\left(\begin{array}{c}
p_{z} \\
\gamma
\end{array}\right) \approx\left(\begin{array}{c}
p_{z 0} \\
\gamma_{0}
\end{array}\right)\left[1+A\left(A+2 p_{x 0}\right) \times\left\{\begin{array}{cc}
\gamma_{\perp 0}^{-2}, & p_{z 0}>0, \\
s / 4 \gamma_{0}^{2}, & p_{z 0}<0 .
\end{array}\right],\right.
$$

where $\gamma_{\perp 0}=\left[1+\left(p_{x 0}\right)^{2}+\left(p_{y 0}\right)^{2}\right]^{1 / 2}, s=-1$ for $p_{z}$, and $s=1$ for $\gamma$. When initially the transverse motion dominates $\left(\left(p_{y 0}\right)^{2},\left(p_{z 0}\right)^{2} \ll\left(p_{x 0}\right)^{2} \gg 1,\left|p_{x 0}\right| \approx \gamma_{0}\right)$, then

$$
\left(\begin{array}{c}
p_{z} \\
\gamma
\end{array}\right) \approx\left(\begin{array}{c}
p_{z 0} \\
\gamma_{0}
\end{array}\right)+\frac{A\left(A+2 p_{x 0}\right)}{2 \gamma_{0}} .
$$

Equations (7)-(11) describe the dynamics of an electron which is determined by the electron initial momentum $\mathbf{p}_{0}$ and its gain in transverse momentum as given by $A\left(\xi_{0}, \xi\right)$.

Let us consider the interaction of an electron with a chirped EM pulse over an infinite interaction region, $\xi_{0}$ $=+\infty$ and $\xi=-\infty$. For definiteness, let us choose a chirped pulse with a Gaussian field envelope and the local frequency varying linearly with the retarded coordinate (linearly chirped pulse),

$$
E_{x}=a_{0} g(\xi) \cos [\Omega(\xi) \xi] .
$$

Here $g(\xi)=\exp \left(-\xi^{2} / \sigma^{2}\right)$ is the field envelope, and $\Omega(\xi)$ $=\omega / \omega_{0}=1+\Delta \Omega \xi / \sigma$ is the normalized local frequency, with $\Delta \Omega=\Omega(\sigma)-1$ being the relative frequency span to characterize the strength of the chirp. For the linearly chirped Gaussian pulse, we obtain

$$
\begin{aligned}
A_{1} \equiv & \int_{+\infty}^{-\infty} E_{x} d \xi=-\frac{\pi^{1 / 2} a_{0} \sigma}{\left(1+\nu^{2}\right)^{1 / 4}} \exp \left[-\frac{\sigma^{2}}{4\left(1+\nu^{2}\right)}\right] \\
& \times \cos \left[\frac{\arctan (\nu)}{2}-\frac{\sigma^{2} \nu}{4\left(1+\nu^{2}\right)}\right],
\end{aligned}
$$

where $\nu \equiv \Delta \Omega \sigma$. Note that the value of $A_{1}$ does not depend on the sign of chirp, $A_{1}(\Delta \Omega)=A_{1}(-\Delta \Omega)$. Figure 1 shows an

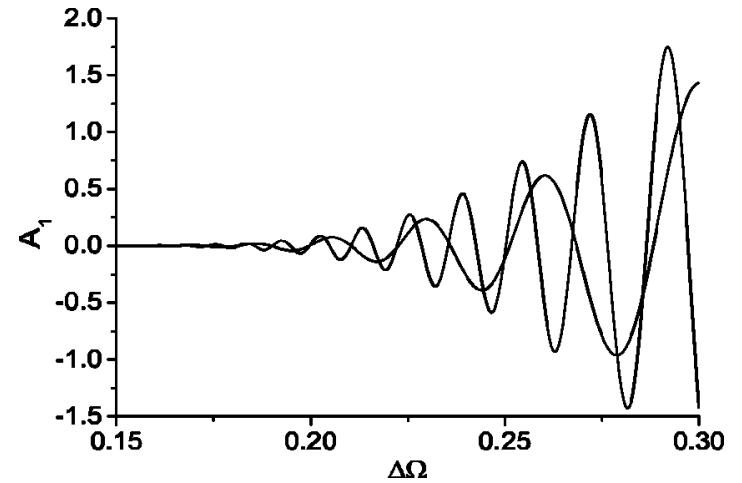

FIG. 1. Transverse momentum gain $A_{1}$ of an electron after interaction with a 1D linearly chirped EM pulse; $\sigma=50$ (the curve with larger period ) and $\sigma=100, a_{0}=1$.

example of the dependence $A_{1}(\Delta \Omega)$, obtained by numerical integration of Eqs. (3) and (5). According to expression (13), when $\sigma^{2} / 4\left(1+\nu^{2}\right) \gg 1$, the momentum (energy) acquired by the electron is small. To increase the value of $A_{1}$, one can choose a shorter pulse or a pulse with a bigger chirp. For a nonchirped pulse $(\Delta \Omega=0) A_{1}=-\pi^{1 / 2} a_{0} \sigma \exp \left(-\sigma^{2} / 4\right)$ and the value of $A_{1}$ is maximum for $\sigma=2^{1 / 2}: A_{1}=-(2 \pi)^{1 / 2}$ $\times \exp (-1 / 2) a_{0}$. The latter expression describes the case of a subcycle EM pulse as studied in Ref. [12]. In contrast to the case of a nonchirped pulse, the momentum (energy) transferred to the electron can be substantial for a long (multiplecycle, $\sigma \gg 1$ ) pulse as well if the chirp is sufficiently strong, so that $\nu^{2} \gg 1$. In this case, we obtain from Eq. (13)

$$
A_{1} \approx-a_{0}\left(\frac{\pi \sigma}{|\Delta \Omega|}\right)^{1 / 2} \exp \left(-\frac{1}{4(\Delta \Omega)^{2}}\right) \cos \left(\frac{\sigma}{4|\Delta \Omega|}-\frac{\pi}{4}\right)
$$

It can be seen that $A_{1}$ is a periodic function with its amplitude and frequency depending on the pulse duration and chirp parameter (see Fig. 1). To illustrate such interaction of an electron with a chirped pulse, we solved Eqs. (3)-(5) numerically for a case when $\Delta \Omega=0.255, a_{0}=3$, and $\sigma=100$; the latter two values approximately correspond to the experimental conditions of Ref. [4]. With these parameters and assuming that $\mathbf{p}_{0}=\mathbf{0}$, we obtain momentum components of $p_{x}=A_{1} \approx 2.19$ and $p_{z}=p_{x}^{2} / 2 \approx 2.39$ after interaction with the linearly chirped pulse. However, when $p_{z 0}=3$ (this corresponds to a kinetic energy of approximately $1.1 \mathrm{MeV}$ ), the transverse momentum dynamics are the same, but the final longitudinal momentum is much higher, $p_{z} \approx 17.71$ $(\approx 8.55 \mathrm{MeV})$. The numerical results show a good agreement with formulas (6)-(11). Note that for nonchirped pulses, the electron momentum is found to be unchanged after interaction.

As follows from Eq. (14), for a linearly chirped pulse the transverse momentum gain $A_{1}$ is exponentially small for a small chirp $(|\Delta \Omega| \ll 1)$. However, for other types of chirp or (and) other pulse envelopes $g(\xi)$ (such pulses can be generated, for example, with spatial light modulators [13]), the result can be qualitatively different. For example, consider an EM pulse with a Gaussian envelope and a nonlinear chirp, 
such that the local frequency has a minimum (maximum) at the pulse center $(\xi=0)$ and is growing (decreasing) linearly to the pulse edges, i.e., $\Omega=1+\Delta \Omega|\xi| / \sigma$. For such a pulse, the value of $A_{1}$ can be calculated analytically when the chirp is small,

$$
A_{1} \approx-4 a_{0} \Delta \Omega / \sigma,
$$

in a good agreement with a numerical integration of expression (8) as well as with numerical solution of Eqs. (3)-(5). Note that Eq. (15) describes a qualitative difference with regard to a linearly chirped pulse, because now $A_{1}$ is inversely proportional to the pulse length. Suppose that the central wavelength in a chirped laser pulse is $0.8 \mu \mathrm{m}, a_{0}$ $=1$ (peak intensity of $\approx 2 \times 10^{18} \mathrm{~W} / \mathrm{cm}^{2}$ ), the full width at half maximum corresponds to $30 \mathrm{fs}$ duration $(\sigma \approx 42.45)$, and $\Delta \Omega=0.1$. Then, from Eqs. (9) and (15), one has $\approx 23 \mathrm{eV}$ for the energy of an electron, initially at rest, after interaction with a nonlinearly chirped pulse. Larger energy gain can be obtained for larger chirp and charges initially copropagating with the pulse, as it is described above.

Consider another case when a chirped pulse possesses an asymmetrical Gaussian envelope, such that $\sigma_{1}\left(\sigma_{2}\right)$ and $\Delta \Omega_{1}$ $\left(\Delta \Omega_{2}\right)$ describe the field when $\xi<0(\xi>0)$. Such asymmetrical envelopes are of interest for various applications and can be generated, for example, by appropriate adjustment of a grating-pair compressor in a femtosecond Ti:sapphire laser [14]. For small chirp, in the case of an asymmetrical EM pulse we have

$$
A_{1} \approx 2 a_{0}\left(\Delta \Omega_{1} / \sigma_{1}-\Delta \Omega_{2} / \sigma_{2}\right) .
$$

Furthermore, in the case of an arbitrary pulse envelope, $g(\xi)$, and an arbitrary chirp, $\Omega(\xi)$, we obtained the following expression for the transverse momentum gain:

$$
A_{1} \approx 2 a_{0} \int_{-\infty}^{+\infty} \frac{d \Omega}{d \xi}\left(3 \frac{d g}{d \xi}+2 \xi \frac{d^{2} g}{d \xi^{2}}\right) d \xi
$$

provided that $g$ and $\Omega$ change on a characteristic time scale much longer than the local wave period and that the chirp is small. To calculate Eq. (17), we divided the electrical field into segments in which the wave phase $\Omega \xi$ changes from $2 \pi n$ to $2 \pi(n+1)$, where $n=0, \pm 1, \pm 2, \ldots$. Then the integral in Eq. (8) was calculated for such a segment expanding $g(\xi)$ and $\Omega(\xi)$ in a Taylor series and keeping only the first derivative of $\Omega$. The value of $A_{1}$ is a sum of the results for all segments. Because the number of segments is large in a multiple-cycle pulse, the sum can be approximated by an integral taking into account that the segment's length depends on the local frequency. This yields the expression (17) from which one can derive Eqs. (15) and (16) by choosing the corresponding pulse envelope and chirp profile. Note that formula (13), which describes an interaction with a linearly chirped pulse, cannot be calculated from Eq. (17); in this case, higher-order derivatives of $\Omega$ should be retained when deriving Eq. (17).

Finally, we discuss the implications of our findings for the case of an electron interaction with a focused (3D) chirped pulse. This is of importance because in experiments, the pulse is usually focused to a small spot size to provide suf-

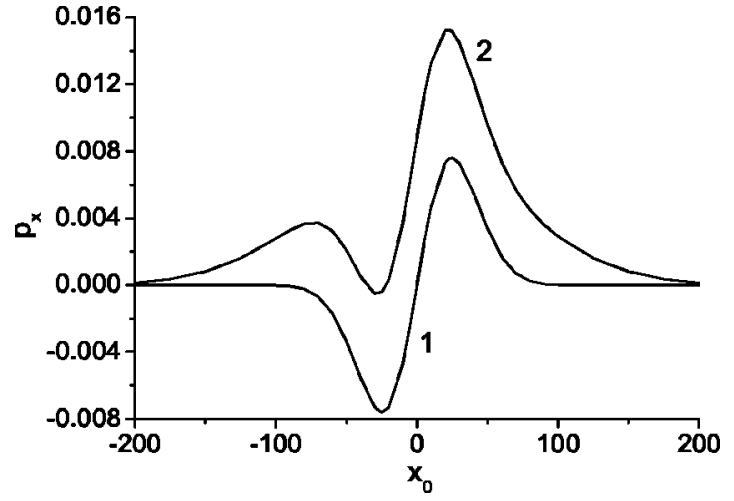

FIG. 2. The transverse momentum of an electron after interaction with a focused nonchirped (curve 1) and a linearly chirped (curve 2) pulse as a function of the initial radial position of the electron; $a_{0}=0.1, \sigma=100, w_{00}=50, \Delta \Omega=-0.213, \mathbf{p}_{0}=0$, and $y_{0}=z_{0}$ $=0$.

ficiently high amplitude. In this 3D case, besides the change of the field in the transverse direction, the longitudinal components of the EM field also need to be considered (see, e.g., Ref. [15]). Furthermore, for a chirped pulse, one also has to take into account that the spot size depends on the local frequency (see, e.g., [16]). To describe the focused EM field, we suppose that before focusing, the pulse radius $w_{1}$ is constant over the pulse length. Then, from the well-known expression for the focused Gaussian beam radius $w=w_{0}(1$ $\left.+z^{2} / Z_{R}^{2}\right)^{1 / 2}$, where $w_{0}$ is the focal radius and $Z_{R}=\omega w_{0}^{2} / 2 c$ is the Rayleigh length, one can see that $w_{0} \sim 1 / \omega[16]$. So, for the focused chirped pulse we can write $w_{0}=w_{00} / \Omega$, where $w_{00}=w_{0}\left(\omega_{0}\right)$. One can see also that because at higher (lower) local frequency the field is focused to a smaller (larger) spot size, the field strength is relatively strong (weak). Indeed, due to the energy conservation, we can write $\left[a_{i n}(\xi) w_{1}\right]^{2}$ $=\left[a(\xi) w_{0}(\xi)\right]^{2}$, where $a_{i n}(\xi)=a_{1} g(\xi)$ and $a(\xi)$ are the local amplitudes before focusing and in focus. Because $w_{1}$ $=$ const and $w_{0} \sim 1 / \Omega$, one finds $a=a_{0} g(\xi) \Omega(\xi), a_{0}$ is a constant. We substituted the latter expression for the field amplitude and $w_{00} / \Omega$ for the spot radius in the well-known expression for the focused monochromatic pulse field [see, e.g., Eqs. (16a)-(16e) in Ref. [15]] to describe the focused chirped-pulse field. This is a good approximation when the characteristic spatial scale for the chirp is much larger than the wavelength. We also neglect a small effect of finite pulse duration [15].

With the described field, choosing $g(\xi)=\exp \left(-\xi^{2} / \sigma^{2}\right)$, we investigated the 3D interaction of an electron with a focused Gaussian chirped pulse by numerical solution of Eq. (1). Figure 2 compares the final transverse momentum $p_{x}$ after interaction with a chirped pulse to that with a nonchirped pulse, in dependence on the initial transverse position of the electron along the $x$ axis (along which the EM field is polarized). One can see that an interaction with a nonchirped pulse leads to the well-known ponderomotive scattering without a transverse momentum gain for on-axis particles, and with a negligible ponderomotive energy gain for electrons with $r_{0}=\left(x_{0}^{2}+y_{0}^{2}\right)^{1 / 2}>2 w_{0}$ [15]. We found also that this scattering does not depend on the polarization of the field 
that agrees with the results of Ref. [15]. However, when the pulse is chirped, there is an energy gain both for initially near-axis particles and for electrons with $r_{0}>2 w_{0}$. In Fig. 2, the value of 50 is chosen for the dimensionless spot radius $w_{00}$ that corresponds to $50 / 2 \pi \approx 8$ wavelengths and is typical for focused high-intensity laser pulses [4]. The results of an electron interaction with a chirped pulse do not change qualitatively when the spot radius is changed. For an inspection of the polarization dependence of chirped pulse interaction, we plot in Fig. 3 the final transverse momentum components as functions of the initial position $y_{0}$ (in the direction perpendicular to the direction of polarization). It can be seen that in this case $p_{y}\left(y_{0}\right)$ is about the same as that for a nonchirped pulse while $p_{x}\left(y_{0}\right)$ (which is equal to zero for a nonchirped pulse) clearly shows the "pure" effect of chirp. Thus, Figs. 2 and 3 show that an electron can gain momentum (energy) from a chirped pulse also in the 3D geometry of focused Gaussian beams, and that this interaction is polarizationdependent. In the 3D case, the longitudinal momentum scales approximately as $p_{x}^{2} / 2$ (see also Ref. [15]).

In summary, it has been shown that a charged particle receives a transverse momentum gain in the direction of polarization and a longitudinal momentum gain related to the transverse one from the interaction with a chirped electromagnetic pulse. This novel effect can influence the interaction between chirped EM pulse and charges qualitatively

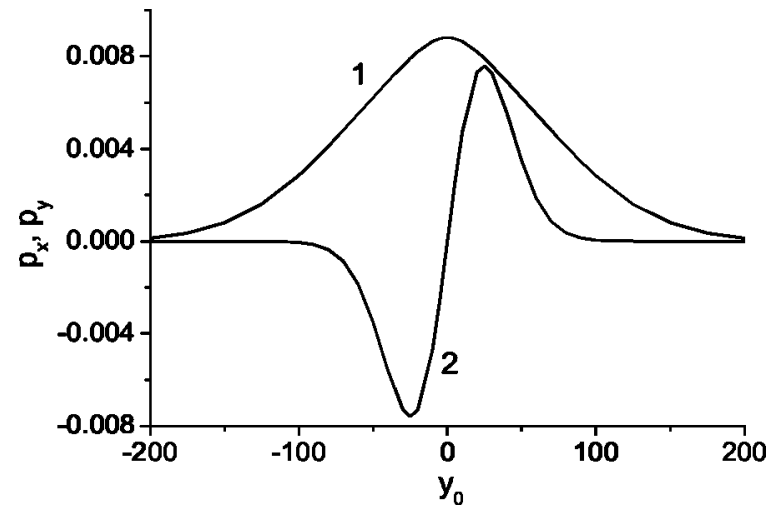

FIG. 3. The final momentum components $p_{x}$ (curve 1) and $p_{y}$ (curve 2) after interaction with a focused chirped pulse in dependence on $y_{0}$. The pulse parameters are the same as in Fig. 2. $\mathbf{p}_{0}$ $=0, x_{0}=z_{0}=0$.

both in 1D and in 3D. When charged particles lose energy after interaction with a chirped EM pulse, the pulse gains energy and this can be used for amplification of the pulse.

This work is supported by the Stichting voor Fundamenteel Onderzoek der Materie (FOM).
[1] W. L. Kruer, The Physics of Laser Plasma Interactions (Addison-Wesley, Reading, MA, 1988).

[2] C. I. Moore, A. Ting, S. J. McNaught, J. Qiu, H. R. Burris, and P. Sprangle, Phys. Rev. Lett. 82, 1688 (1999).

[3] See, e.g., recent articles: Y. I. Salamin, G. R. Mocken, and C. H. Keitel, Phys. Rev. ST Accel. Beams 5, 101301 (2002); F. He et al., Phys. Rev. E 68, 046407 (2003).

[4] G. Malka, E. Lefebvre, and J. L. Miquel, Phys. Rev. Lett. 78, 3314 (1997).

[5] J. Faure et al., Phys. Rev. E 63, 065401 (2001).

[6] T. Brabec and F. Krausz, Rev. Mod. Phys. 72, 545 (2000).

[7] D. F. Gordon et al., Phys. Rev. Lett. 90, 215001 (2003).
[8] R. Hajima and R. Nagai, Phys. Rev. Lett. 91, 024801 (2003).

[9] J. M. Dias et al., Phys. Rev. Lett. 78, 4773 (1997).

[10] A. S. Il'in, V. V. Kulagin, and V. A. Cherepenin, Plasma Phys. Rep. 27, 1048 (2001).

[11] F. V. Harteman et al., Phys. Rev. E 51, 4833 (1995).

[12] B. Rau, T. Tajima, and H. Hojo, Phys. Rev. Lett. 78, 3310 (1997).

[13] A. M. Weiner, Rev. Sci. Instrum. 71, 1929 (2000).

[14] Cs. Toth et al., Opt. Lett. 28, 1823 (2003).

[15] B. Quesnel and P. Mora, Phys. Rev. E 58, 3719 (1998).

[16] A. E. Siegman, Lasers (University Science Books, Mill Valley, CA, 1986), p. 675 